GPU-accelerated voxelwise hepatic perfusion quantification

This article has been downloaded from IOPscience. Please scroll down to see the full text article.

2012 Phys. Med. Biol. 575601

(http://iopscience.iop.org/0031-9155/57/17/5601)

View the table of contents for this issue, or go to the journal homepage for more

Download details:

IP Address: 141.211.173.82

The article was downloaded on 25/06/2013 at 20:35

Please note that terms and conditions apply. 


\title{
GPU-accelerated voxelwise hepatic perfusion quantification
}

\author{
H Wang $^{1}$ and Y Cao ${ }^{1,2,3}$ \\ ${ }^{1}$ Departments of Radiation Oncology, University of Michigan, Ann Arbor, MI, 48109, USA \\ 2 Departments of Radiology, University of Michigan, Ann Arbor, MI, 48109, USA \\ ${ }^{3}$ Departments of Biomedical Engineering, University of Michigan, Ann Arbor, MI, 48109, USA \\ E-mail: hesheng@umich.edu
}

Received 9 May 2012, in final form 27 June 2012

Published 14 August 2012

Online at stacks.iop.org/PMB/57/5601

\begin{abstract}
Voxelwise quantification of hepatic perfusion parameters from dynamic contrast enhanced (DCE) imaging greatly contributes to assessment of liver function in response to radiation therapy. However, the efficiency of the estimation of hepatic perfusion parameters voxel-by-voxel in the whole liver using a dual-input single-compartment model requires substantial improvement for routine clinical applications. In this paper, we utilize the parallel computation power of a graphics processing unit (GPU) to accelerate the computation, while maintaining the same accuracy as the conventional method. Using compute unified device architecture-GPU, the hepatic perfusion computations over multiple voxels are run across the GPU blocks concurrently but independently. At each voxel, nonlinear least-squares fitting the time series of the liver DCE data to the compartmental model is distributed to multiple threads in a block, and the computations of different time points are performed simultaneously and synchronically. An efficient fast Fourier transform in a block is also developed for the convolution computation in the model. The GPU computations of the voxel-by-voxel hepatic perfusion images are compared with ones by the CPU using the simulated DCE data and the experimental DCE MR images from patients. The computation speed is improved by 30 times using a NVIDIA Tesla C2050 GPU compared to a $2.67 \mathrm{GHz}$ Intel Xeon CPU processor. To obtain liver perfusion maps with 626400 voxels in a patient's liver, it takes 0.9 min with the GPU-accelerated voxelwise computation, compared to $110 \mathrm{~min}$ with the $\mathrm{CPU}$, while both methods result in perfusion parameters differences less than $10^{-6}$. The method will be useful for generating liver perfusion images in clinical settings.
\end{abstract}

(Some figures may appear in colour only in the online journal) 


\section{Introduction}

The liver has a dual-blood supply: one is from the hepatic artery and another from the portal vein. In the normal liver parenchyma, portal venous perfusion contributes to $70-80 \%$ of the total perfusion, and hepatic arterial perfusion about 20-30\% (Chiandussi et al 1968). Diseases and tumors in the liver can alter both portal venous and hepatic arterial perfusion in the tissue (Hashimoto et al 2006, Leggett et al 1997, Van Beers et al 2001). Quantitative arterial and portal venous perfusion derived from dynamic contrast enhanced (DCE) imaging by fitting a pharmacokinetic model has shown clinical values for detection of hepatic cancers, diagnosis of liver cirrhosis and its severity, and assessment of therapy effectiveness (Thng et al 2010, Materne et al 2002, Goetti et al 2011). Recently, Cao et al (2007, 2012) show volumetric hepatic perfusion images derived from DCE-CT or DCE-MRI have the potential to assess global and spatial liver function change in response to radiation therapy. To quantify both arterial and hepatic venous perfusion, a dual-input single-compartment model is commonly used to fit the DCE data by minimizing a multivariable nonlinear least-squares (NLS) cost function (Dawson 2007, Materne et al 2002, Thng et al 2010). As MRI and CT technologies are advancing, it is possible to acquire volumetric liver DCE images with both high spatial and temporal resolutions. However, fitting the volumetric liver DCE images to the kinetic model voxel-by-voxel is very time consuming, which hinders utilization of the volumetric hepatic perfusion measurements in clinical applications.

Several studies have proposed computationally efficient methods to fit the liver DCE data to the pharmacokinetic model (Cao et al 2007, Hagiwara et al 2008, Materne et al 2002, Pandharipande et al 2005). The methods linearize the kinetic model by directly using either the derivative of the liver DCE time-concentration curve or the integrals of the two blood input functions, and then estimate the perfusion parameters by a linear least-squares (LLS) fitting. Although the LLS methods speed up the perfusion estimation by up to ten times compared with the conventional NLS method (Murase et al 2007), a LLS fitting can generate biased results even though the data noise is white (Feng et al 1996). Also, time delays of the contrast agent (CA) bolus arrival from the artery and portal vein to the liver parenchyma, which can have a great impact on the perfusion estimation (Miyazaki et al 2008), have been either ignored or only partially considered in the LLS models (Murase et al 2007, Cao et al 2006).

A graphics processing unit (GPU), originally designed for graphic rendering, possesses a great arithmetic capability, and is well suited for computationally intensive, highlyparallelizable applications (Owens et al 2008). Recent advances in the GPU programming architecture have enabled GPU for a variety of computations in science and medicine (Jia et al 2011, Pratx and Xing 2011). Therefore, an alternative to accelerating voxelwise hepatic perfusion quantification is to utilize the GPU. In this paper, we implemented compute unified device architecture (CUDA)-based parallel computation to fit DCE MR images with the liver dual-input single-compartment model in order to estimate volumetric hepatic perfusion maps. Our parallel computation strategy can be generalized, and several components of our programs can be directly adopted for fitting DCE images to other pharmacokinetic models.

\section{Methods and materials}

\subsection{Pharmacokinetic model of hepatic perfusion}

Liver parenchyma receives a dual blood supply from the hepatic artery and the portal vein, and then it drains blood into the central vein. A dual-input single compartment model that is 

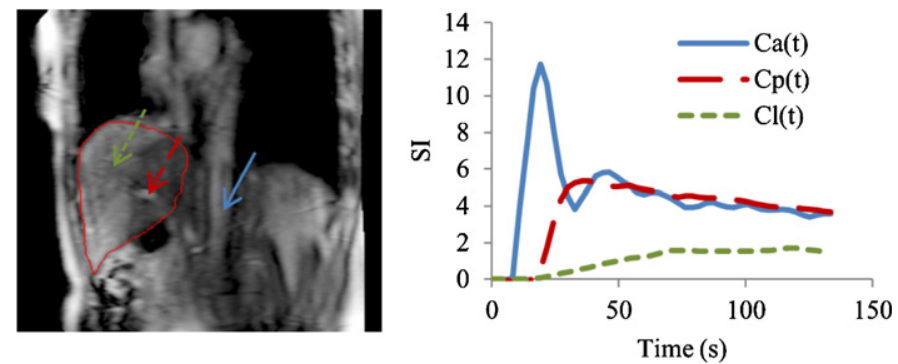

Figure 1. Left: a sagittal MRI slice that covers VOIs to determine arterial input $\left(C_{a}\right)$, portal venous input $\left(C_{p}\right)$ and time-course signals of liver tissue $\left(C_{l}\right)$. Right: time-concentration curves of $C_{a}, C_{p}$ and $C_{l}$. Blue Arrow: aorta; red arrow: portal vein; green arrow: liver tissue.

commonly used to describe the pharmacokinetics and fit DCE data is expressed as follows (Cao et al 2006, Materne et al 2002):

$$
\frac{\mathrm{d} \bar{C}_{l}(t)}{\mathrm{d} t}=k_{a} C_{a}\left(t-\tau_{a}\right)+k_{p} C_{p}\left(t-\tau_{p}\right)-k_{l} \bar{C}_{l}(t)
$$

where $C_{a}$ and $C_{p}$ are CA concentrations of respective artery and portal vein; $\bar{C}_{l}$ is the modeled $\mathrm{CA}$ concentration at liver parenchyma where the acquired CA concentration is denoted as $C_{l}$; $k_{a}, k_{p}$ and $k_{l}$ are arterial inflow, portal venous inflow and central venous outflow rate constants, respectively; and $\tau_{a}$ and $\tau_{p}$ are time delays of the CA bolus arrival from the artery and portal vein, respectively, to the parenchyma. The differential equation (1) is solved as

$$
\bar{C}_{l}(t)=\int_{0}^{t}\left(k_{a} C_{a}\left(\tau-\tau_{a}\right)+k_{p} C_{p}\left(\tau-\tau_{p}\right)\right) \mathrm{e}^{-k_{l}(t-\tau)} \mathrm{d} \tau=f(t) \otimes h(t),
$$

where we denote $f(t)=k_{a} C_{a}\left(t-\tau_{a}\right)+k_{p} C_{p}\left(t-\tau_{p}\right)$ and $h(t)=\mathrm{e}^{-k_{l} t}$, and $\otimes$ is a convolution operator.

To estimate perfusion parameters $k_{a}, k_{p}, k_{l}, \tau_{a}$ and $\tau_{p}$, time-concentration curves $C_{l}, C_{a}$ and $C_{p}$ are derived from DCE images (figure 1) and fitted to the kinetic model in equation (2) by minimizing a NLS cost function $E$ between the acquired $C_{l}$ and modeled $\bar{C}_{l}$ :

$$
E=\sum_{i=0}^{N_{t}-1}\left(C_{l}(i T)-f(i T) \otimes h(i T)\right)^{2},
$$

where $T$ is a sampling time interval of DCE imaging, and $N_{t}$ is a total number of time points acquired during dynamic imaging. The NLS minimization with respect to the five parameters $\left(k_{a}, k_{p}, k_{l}, \tau_{a}, \tau_{p}\right)$ is commonly solved by the Nelder-Mead 'Simplex' optimization algorithm (Lagarias et al 1998, Nelder and Mead 1965). The Simplex algorithm is an iterative optimization procedure that heuristically updates the solution according to the cost function values at the previous iterations. Given that in our study the number of voxels in the liver MR images of the patients with intrahepatic cancers is as large as $10^{6}$, it is very time consuming to estimate hepatic perfusion voxel-by-voxel throughout the whole liver.

\subsection{GPU-accelerated perfusion quantification}

We aimed to accelerate volumetric hepatic perfusion quantification without compromising accuracy by using a GPU. To maximally utilize the GPU, computation should be structured to expose as much parallelization as possible (Owens et al 2008). Two types of computations 
in the volumetric DCE quantification can be parallelized: one is to simultaneously estimate perfusion among the $N_{v}$ voxels, and another is to concurrently compute the cost function values from the $N_{t}$ time point curves in one voxel. Synchronization of the $N_{t}$ time point parallel computation is required in order to obtain a cost function value per voxel (equation (3)). On the other hand, parallel computations for multiple voxels should be asynchronous to allow individual convergence behaviors in the Simplex minimization of the voxels since the voxels are independent of each other. In the following subsections, we describe the implementations of these two parallelization strategies on the GPU using CUDA from NVIDIA (Nvidia 2010).

2.2.1. Introduction of GPU and CUDA. A GPU device is comprised of a number of multiprocessors (MPs), each of which consists of multiple streaming processors, or processor cores, for massive parallel computation. CUDA abstracts the complexity in programming the graphic hardware, and provides a general-purpose parallel computing platform (Nvidia 2010). In CUDA, multiple copies of a $\mathrm{C} / \mathrm{C}++$ function, called a kernel, are executed as parallel threads on the GPU, multiple threads are scaled as a block, and all the blocks that are designed for a specific task compose a grid. The threads can access a large amount of, but slow, off-chip device memory, a limited amount of, but fast, on-chip shared memory, a constant cache, and a texture cache. Therefore, developing a CUDA GPU-accelerated application involves both the partition of computation units into parallel executions in a hierarchical grid-block-thread configuration for maximal parallelization, and the explicit management of memory access for optimal performance.

2.2.2. Parallelization of multivoxel perfusion computation. In CUDA, the threads in a block can share data through the shared memory and synchronize their kernel executions, but there is no explicit support for the synchronization of threads across different blocks. Therefore, we configure $N_{v}$ blocks for parallel computations for $N_{v}$ voxels, and one block exclusively performs computation for one voxel. The computations across the blocks are concurrent but independent. The following subsections describe how the NLS fitting for one voxel is performed on the threads of a block.

2.2.3. Parallelization of the NLS cost function calculation. The perfusion quantification at each voxel is to minimize the NLS cost function of equation (3) by the Simplex algorithm. The cost function (equation (3)) calculations include (1) a convolution of two time curves ( $f(i T)$ and $h(i T)$ ) with $N_{t}$ time points of each; (2) a square of the difference between the measured and modeled values at each time point; and (3) a sum of the square differences over $N_{t}$ time points. The most expensive computation is the convolution if it is straightforwardly computed, which requires every time point of $f(i T)$ to be multiplied by every point of $h(i T)$. Alternatively, the time-domain convolution can be computed in the Fourier domain, in which the convolution becomes a complex-number multiplication at each frequency component. Therefore, Fourier transform (FT) and its inverse transform have to be implemented on a GPU block in order to be called upon to calculate the cost function value at each of the Simplex iterations on the block. Although CUDA provides a parallel fast Fourier transform library (CUFFT), the CUFFT can only be called by the host CPU to perform transforms of multiple-batch data on the whole GPU but not on a block. Therefore, we developed a Block-FFT to perform the FT in order to execute the Fourier domain convolution on a block.

Block-FFT. First, a time curve with $N_{t}$ time points is zero-padded to have $N_{t}=q p$ (i.e. $q \times p$ ) points, where $p$ is a power of $2, q$ is a small integer greater than 1 , and $N_{t}$ is as close to the number of the original time points as possible. To avoid the circular convolution problem due 
(a) Time Point Configuration

\begin{tabular}{c|c|c|c|c|c}
\hline$\left(i_{1}, i_{2}\right)$ & 0 & 1 & 2 & $\ldots$ & $p-1$ \\
\hline 0 & 0 & 1 & 2 & $\ldots$ & $p-1$ \\
\hline$\ldots$ & $\ldots$ & $\ldots$ & $\ldots$ & $\ldots$ & $\ldots$ \\
\hline$q-1$ & $(q-1) p$ & $(q-1) p+1$ & $(q-1) p+2$ & $\ldots$ & $q p-1$ \\
\hline$q$ & $q p$ & $q p+1$ & $q p+2$ & $\ldots$ & $(q+1) p-1$ \\
\hline$\ldots$ & $\ldots$ & $\ldots$ & $\ldots$ & $\ldots$ & $\ldots$ \\
\hline $2 q-1$ & $(2 q-1) p$ & $(2 q-1) p+1$ & $(2 q-1) p+2$ & $\ldots$ & $2 q p-1$ \\
\hline
\end{tabular}

(b) Frequency Component Configuration

\begin{tabular}{c|c|c|c|c|c}
\hline$\left(k_{l}, k_{2}\right)$ & 0 & 1 & 2 & $\ldots$ & $p-1$ \\
\hline 0 & 0 & $2 q$ & $4 q$ & $\ldots$ & $2 q(p-1)$ \\
\hline$\ldots$ & $\ldots$ & $\ldots$ & $\ldots$ & $\ldots$ & $\ldots$ \\
\hline$q-1$ & $q-1$ & $3 q-1$ & $5 q-1$ & $\ldots$ & $q(2 p-1)-1$ \\
\hline$q$ & $q$ & $3 q$ & $5 q$ & $\ldots$ & $q(2 p-1)$ \\
\hline$\ldots$ & $\ldots$ & $\ldots$ & $\ldots$ & $\ldots$ & $\ldots$ \\
\hline $2 q-1$ & $2 q-1$ & $4 q-1$ & $6 q-1$ & $\ldots$ & $2 q p-1$ \\
\hline
\end{tabular}

Figure 2. Configurations of time points (a) and frequency components (b) designed for the BlockFFT. Both tables contain $2 q$ rows and $p$ columns. $i_{l}$ and $k_{l}$ are row indices and $i_{2}$ and $k_{2}$ are column indices for the time and frequency component table, respectively. Time points in rows $q+1$ to $2 q-1$ (shaded) are zero due to zero padding. The frequency components in shaded rows $q+1$ to 2q-1 can be determined by complex conjugation of real signal FT.

to the Fourier domain multiplication (Oppenheim et al 1999), the curve is further zero-padded to $2 N_{t}$ points. Next, we organize $2 N_{t}$ points of the curve into a $2 q \times p$ table (row $\times$ column) and order them along the row first (figure 2(a)), where the temporal index $i$ of the curve is rewritten by two dimensional (2D) indices as $i=p i_{1}+i_{2}\left(0 \leqslant i_{1} \leqslant 2 q-1,0 \leqslant i_{2} \leqslant p-1\right)$. According to the general Cooley-Tukey algorithm (Duhamel and Vetterli 1990), FT of a curve (f) with $2 N_{t}$ points can be written as

$$
F_{k}=F_{2 q k_{2}+k_{1}}=\sum_{i_{2}=0}^{p-1}\left\{W_{2 N_{t}}^{i_{2} k_{1}}\left(\sum_{i_{1}=0}^{2 q-1} f_{p i_{1}+i_{2}} W_{2 q}^{i_{1} k_{1}}\right)\right\} W_{p}^{i_{2} k_{2}}
$$

where $W_{P}^{Q}=\exp (-j 2 \pi Q / P)$ for integers $P$ and $Q$, called a twiddle factor in FT, and $k(0 \leqslant k \leqslant$ $\left.2 N_{t}-1\right)$ is a frequency index and also expressed by $2 \mathrm{D}$ indices as $k=2 q k_{2}+k_{1}\left(0 \leqslant k_{1} \leqslant\right.$ $\left.2 q 1,0 \leqslant k_{2} \leqslant p-1\right)$. Using the configuration of the $2 q \times p$ table in figure 2(a), FT of curve $f$ in the above equation can be done by performing a $2 q$-point FT along each column (inner parenthesis), and finally performing a $p$-point FT along each row (outer parenthesis). As a result, the frequency components during the FT can be stored in a $2 q \times p$ complex-number table as in figure 2(b), in which the frequency components are ordered along the column first.

To further speed up computation and reduce memory usage, the complex conjugation of FT of the real data is used. By organizing the frequency components into the $2 q \times p$ frequency table as in figure 2(b), $F_{2 q k_{2}+k_{1}}=F_{2 q\left(p-1-k_{2}\right)+\left(2 q-k_{1}\right)}^{*}$, and thus, computation and data storage for the frequency components from row $q+1$ to $2 q-1$ can be omitted. In addition, the time points of the curve from row $q$ to $2 q-1$ in the time table figure 2(a) are zeros (due to zero padding) and do not have to be stored. As a result, $(q+1) \times p$ memory are allocated on the shared memory of a block for the $(q+1) \times p$ complex frequency components (rows 0 to $q$ of figure 2(b)). Rows 0 to $q-1$ of the real part of the allocated memory are also used to load the $N_{t}$ time points of a time curve before its FT. In our CUDA codes, a block is configured to 


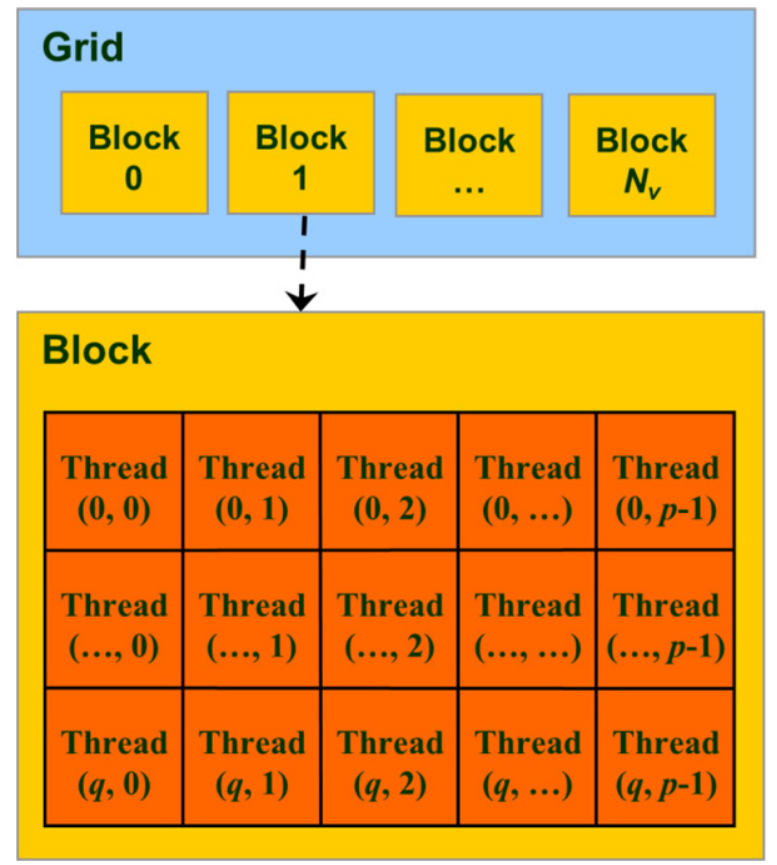

Figure 3. CUDA thread-block-grid hierarchy for the Block-FFT. The threads in a block are indexed with $\left(k_{l}, k_{2}\right)$ used in figure $2(\mathrm{~b})$, and so one-to-one corresponds to the frequency component computation.

have $(q+1) \times p$ threads as a 2D matrix (figure 3 ), each of which executes the computation for one frequency component in the table memory figure 2(b).

With one-to-one correspondences of the table memory cells and threads, the Block-FFT performs calculations on each thread as follows: (1) compute $\sum_{i_{1}=0}^{q-1} f_{p i_{1}+i_{2}} W_{2 q}^{i_{1} k_{1}}$ along the column with a total of $q$ points; (2) multiple $W_{2 N_{t}}^{i_{2} k_{1}}$ to the column-FT results in each cell $\left(k_{1}, k_{2}\right)$ of the table; and (3) perform FFT along the row with a total of $p$ points. In the third step, the recursive radix-2 FFT algorithm (Moreland and Angel 2003) is utilized since $p$ (the number of components in a row) is a power of 2 . As a total, each thread performs $q+1+\log _{2}(p)$ complex-number multiplications and $q+\log _{2}(p)$ complex-number additions to perform the FT of a $2 q p$-point curve. Of note, $q$ is designed to be a small integer; thus the $q$-point FT would not slow down the Block-FFT, but $q$ is greater than 1 in order to utilize the complex conjugation relationship of the frequency components between the rows in the frequency table. Using the same principle, the inverse Block-FFT is implemented by inversing the three-step process and replacing the twitter factor with its complex conjugate, and then dividing the real components of the results by the number of points.

$N L S$ cost function calculation. The NLS cost function calculation at each trial solution of $\left(k_{l}\right.$, $\left.k_{a}, k_{p}, \tau_{a}, \tau_{p}\right)$ during the Simplex searching process involves three time-concentration curves. The curves $C_{a}$ and $C_{p}$, used by all the voxels, are loaded into the texture memory and accessed by all the blocks. Each of the liver dynamic curves $C_{l}$ after zero padding to $N_{t}$ is stored as a contiguous segment in the device memory to allow the threads in a block to efficiently access the coalescent data. 
Table 1. Operation counts for convolutions in time-domain, and frequency-domain ${ }^{\mathrm{a}}$ on the $\mathrm{CPU}$ and GPU.

\begin{tabular}{llll}
\hline Operations & $\begin{array}{l}\text { Time-domain } \\
\text { convolution }\end{array}$ & $\begin{array}{l}\text { Frequency-domain } \\
\text { convolution on } \mathrm{CPU}^{\mathrm{b}}\end{array}$ & $\begin{array}{l}\text { Frequency-domain } \\
\text { convolution on GPU }\end{array}$ \\
\hline $\begin{array}{l}\text { Complex } \\
\text { multiplications }\end{array}$ & & $p(q+1)\left(2 \log _{2} p+2 q+3\right)$ & $2 \log _{2} p+2 q+3$ \\
$\begin{array}{l}\text { Complex } \\
\text { Additions }\end{array}$ & & $p(q+1)\left(2 \log _{2} p+2 q\right)$ & $2 \log _{2} p+2 q$ \\
$\begin{array}{l}\text { Real } \\
\text { Multiplications }\end{array}$ & $p q(p q+1) / 2$ & $4 p(q+1)\left(2 \log _{2} p+2 q+3\right)$ & $4\left(2 \log _{2} p+2 q+3\right)$ \\
$\begin{array}{l}\text { Real } \\
\text { Additions }\end{array}$ & $p q(p q-1) / 2$ & $2 p(q+1)\left(4 \log _{2} p+4 q+3\right)$ & $2\left(4 \log _{2} p+4 q+3\right)$ \\
\hline
\end{tabular}

${ }^{\mathrm{a}}$ Frequency-domain convolution includes FFT, frequency component multiplication and inverse FFT.

${ }^{\mathrm{b}}$ The FFT on CPU is based upon the Cooley-Tukey algorithm.

${ }^{c}$ The Block-FFT is utilized for convolution on the GPU. The counts are operations on each thread.

To compute the convolution $f(i T) \otimes h(i T)$ in the cost function, $C_{a}(i T)$ and $C_{p}(i T)$ are read from the texture memory, linearly interpolated according to the given time delays $\left(\tau_{a}, \tau_{p}\right)$, summed into $f(i T)$, and maintained in the frequency component table in the share memory. Its Fourier transform $F(k)$ is obtained by performing the Block-FFT as described in the previous subsection. Then, the analytical form of FT of $h(i T)$ after zero-padding to $2 N_{t}$ points, $H(k)=\frac{1-(-1)^{k} \mathrm{e}^{-k_{l} N_{T} T}}{1-\mathrm{e}^{-k_{l} T} W_{2 N_{t}}}$, is multiplied by $F(k)$ in each cell of the frequency component table according to their frequency correspondences. Finally, the convolution result $\bar{C}_{l}(i T)$ is obtained by the inverse Block-FFT. Table 1 shows the operation counts per thread for the convolution on the GPU, as well as the operation numbers of a $\mathrm{CPU}$ for the time-domain and frequency-domain convolutions.

After each thread calculates the squared distance between $\bar{C}_{l}(i T)$ and $C_{l}(i T)$ at a time point $i$, the cost function value is a summation of the squared distances over all the time points in the table memory cells using a naïve parallel scan algorithm executed on the block (Daniel 2005).

2.2.4. Implementation of the simplex algorithm on GPU. The Nelder-Mead Simplex method is a direct search method to minimize a scalar-valued nonlinear function of $n$ real variables using function values only. In the Simplex method, a vector of the $n$ variables of a possible solution is defined as a vertex. The search begins with a simplex with $n+1$ vertices and the associated function values, and then one new vertex is calculated and tested. The search is continued until reaching stopping criteria. In our NLS cost function, there are five unknown parameters $\left(k_{l}, k_{a}, k_{p}, \tau_{a}, \tau_{p}\right)$. Thus, a six-vertex simplex plus an extra vertex as the new solution are maintained and updated based on their cost function values by following the Simplex searching scheme. For fast access, the memories for the seven vertices and their cost function values are allocated in the shared memory. The Simplex search scheme in the NLS fitting is implemented on a block and exactly as the one reported by Lagarias et al (1998). Branching to a new vertex in the Simplex searching is only based on the cost function values of the vertices, and all the threads in a block follow the same branching path. Figure 4 shows the flowchart of the GPU-accelerated voxelwise perfusion quantification.

\subsection{GPU and CPU implementations}

The GPU computation was implemented by GNU C with a CUDA 4.0 toolkit on an NVidia Tesla C2500 GPU card. The card has 14 MPs and each MP has 32 cores. The host computer has 
Determine $q$ and $p$ according to the number of time points of DCE data

Load DCE data of $N_{v}$ liver voxels from CPU to GPU

Configure block and thread numbers for GPU computation

Execute the kernel of perfusion estimations on blocks in parallel

Initialize a Simplex by given initial values

Calculate the cost function value of each vertex of the Simplex

Update the simplex by the Simplex searching scheme

Repeat updating the simplex until reaching termination criteria

Output the estimated perfusion parameters of $N_{v}$ voxels from GPU to CPU

Load additional voxels of DCE data to GPU until computations are done for all voxels

Figure 4. Flowchart of the GPU-accelerated voxelwise hepatic perfusion estimation.

a 6-core 2.67 GHz Intel Xeon CPU X5650. The NLS fitting of the DCE images requires a highprecision cost function calculation. Therefore, double-precision computation and memory allocation were used on the GPU. For the purpose of comparison, the conventional hepatic perfusion computation was implemented on the host CPU by using GNU C programming and named as C-CPU. The C-CPU method performed voxel-by-voxel perfusion computation which minimized equation (3) without parallelization. The Simplex algorithm in the CPU method used the same search scheme as Lagarias et al (1998), adopted from Numeric Recipes 3.0 (Press et al 2007). Convolution in the cost function was also calculated in the Fourier domain by using the FFT in the GNU Scientific Library (GSL) (Galassi et al 2009). Both the CPU and GPU computations started with the same initial solution and were terminated when a difference between the maximal and minimal function values of the vertices in the simplex was less than $10^{-8}$ or the iteration number was greater than 600 .

In order to evaluate the efficiency of GPU computation, computation time per voxel was calculated by averaging the total computation time over the same number of voxels for the GPU and C-CPU methods. The total time for the GPU computation included the time to load the DCE data into the GPU device memory from the host CPU memory, estimating perfusion parameters over a given number of voxels on the GPU, and exporting the resultant perfusion parameters to the CPU memory. In the C-CPU computation, only the voxel-by-voxel perfusion estimation time was included.

\subsection{Evaluation experiments}

2.4.1. Simulation studies. Simulated data with known perfusion parameters were used to evaluate the accuracy and efficiency of liver perfusion quantification on the GPU. We obtained an arterial input $C_{a}$ and a portal venous input $C_{p}$ from our previous IRB-approved DCE-CT study (Cao et al 2006, 2007), in which the DCE data was acquired at a temporal resolution of $1 \mathrm{~s}$ for $2 \mathrm{~min}$. The curves $C_{a}$ and $C_{p}$ were interpolated to obtain curves with the following numbers of time points: $32,64,128,256,320$ and 448. A time-concentration curve $C_{l}$ was simulated from $C_{a}$ and $C_{p}$ using equation (2), where $k_{a}, k_{p}$ and $k_{l}$ were set to be 20, 100 and $400 \mathrm{ml} / 100 \mathrm{~g} / \mathrm{min}$, respectively, and $t_{a}$ and $t_{p}$ were $1 \mathrm{~s}$ and $2 \mathrm{~s}$, respectively. These parameters 
are typical for normal hepatic perfusion (Cao et al 2006). The $C_{l}$ curve was replicated up to 10000 voxels. The perfusion parameters of these voxels were estimated in parallel on the GPU, and in serial on the host CPU. Both computations were initialized with $10 \mathrm{ml} / 100 \mathrm{~g} / \mathrm{min}$, $80 \mathrm{ml} / 100 \mathrm{~g} / \mathrm{min}, 200 \mathrm{ml} / 100 \mathrm{~g} / \mathrm{min}, 2 \mathrm{~s}$ and $3 \mathrm{~s}$ for $k_{a}, k_{p}, k_{l}, \tau_{a}$ and $\tau_{p}$, respectively. The computation time per voxel with respect to the number of voxels $\left(N_{v}\right)$ and the number of time points $\left(N_{t}\right)$ were measured for both the GPU and C-CPU computations.

To evaluate the accuracy of the GPU perfusion computation, Gaussian noise was added to the simulation data. The noise is quantified by the contrast-to-noise ratio (CNR) that was defined as the ratio of the signal peak to the standard deviation of the curve at the baseline. We used the typical CNR of 150 and 100 for $C_{a}$ and $C_{p}$, respectively, and varied the CNRs of $C_{l}$ from 10 to 100 by steps of 10 . For each tested CNR, the noisy signals with 128 time points were generated 1000 times. The perfusion parameters were estimated by both the GPU and C-CPU methods, as well as the method implemented in Matlab on the CPU, named as MatlabCPU, using the Matlab optimization function 'fminsearch'. The function 'fminsearch' used the Simplex search algorithm in exactly the same way as our GPU and C-CPU computations. The means and standard deviations of $k_{a}, k_{p}$ and $k_{l}$ estimated by the three methods were compared with respect to the noise levels.

We also evaluated the speed of the Block-FFT by comparing it with the CUFFT of multiple liver curves on the GPU. First, the curves were zero padded to double the number of points in order to perform convolution computation in Fourier domain. Then, the zero-padded curves $C_{l}$ of $N v$ voxels were Fourier transformed and then inverse Fourier transformed on the GPU using the Block-FFT and the CUFFT. Both computations were invoked in the same way by the CPU for parallel FT of the $N_{v}$ voxels. The CUFFT performed the transformation by its realto-complex Fourier transform and complex-to-real inverse Fourier transform. We compared the time per curve transform between the two implementations with respect to the number of curves $\left(N_{v}\right)$ and the number of time points in the curve $\left(N_{t}\right)$. To evaluate the accuracy of the Block-FFT, a root mean squared error (RMSE) of a curve transformed by forward FT and followed by inverse FFT to the original curve was calculated.

2.4.2. Patients hepatic perfusion studies. DCE-MRI scans of three patients with intra-hepatic cancers were obtained in a prospective IRB-approved protocol. The DCE data were acquired during a bolus injection of $15 \mathrm{ml} \mathrm{Gd-DTPA}$ at a rate of $2 \mathrm{ml} \mathrm{s}^{-1}$ on a clinical 3T MR scanner (Philips Achieva 3.0T; Philips Healthcare, Netherlands). A 3D DCE MRI covering the whole liver was obtained every $2.37 \mathrm{~s}$ for a total of $2 \mathrm{~min}$ with a gradient echo pulse sequence (TR/TE/FA: 4.48/2.15/20'; Matrix: $320 \times 320 \times 66$; FOV: $33 \times 33 \times 19.8 \mathrm{~cm}$; SENSE factor: 2 in 2 different directions). The 3D data was acquired in sagittal/coronal orientation to avoid the inflow effect.

An arterial input $\left(C_{a}\right)$ and a portal venous input $\left(C_{p}\right)$ were obtained from VOIs of the aorta and portal vein, respectively, on the MR images. The voxel numbers of the aorta and portal vein VOIs were approximately 800 and 400, respectively. The MR time-intensity signals were converted to the CA time-concentration curves by assuming a linear relationship between the CA concentration and the relaxivity (R1) enhancement after the CA administration. As the original DCE data had 42-48 temporal phases, we attached zero-intensity phases to the ends of the time series to have 48 phases (i.e. $N_{t}=48$ ). The GPU and C-CPU methods were applied to estimate volumetric perfusion maps from the DCE data. In the GPU computation, $N_{t}$ of 48 was decomposed to be $3 \times 16(q \times p)$ so that a GPU block was configured to have $4 \times 16((q+1) \times p)$ threads. According to availability of the device memory, the liver dynamic curves from the first 60000 voxels were loaded into the device memory, and the GPU was configured into 60000 blocks for parallel perfusion quantification over the 60000 

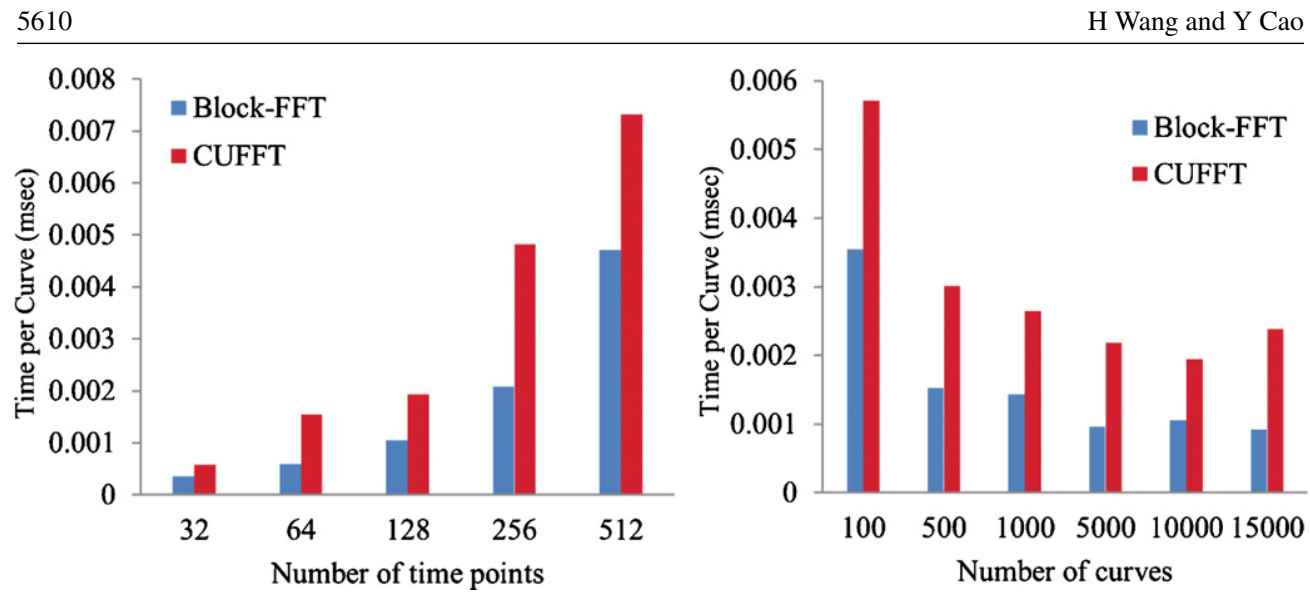

Figure 5. Performances of the Block-FFT and CUFFT of time curves with $N_{t}$ time points and over $N_{v}$ voxels. Left: computation time per curve transform versus the number of time points over $N_{v}=10000$ voxels. Right: computation time per curve transform versus the number of voxels for a curve with 128 time points.

voxels. After completing the first 60000 -voxel computations, the liver data of the next 60000 voxels were loaded into the GPU for perfusion estimation. The process was stopped when computations for all the voxels in the liver volume were done. The absolute differences of perfusion parameters between GPU and C-CPU computations were calculated at each voxel to evaluate the accuracy of the GPU computation.

\section{Results}

\subsection{Simulation studies}

The comparison of the computation speeds of the Block-FFT and the CUFFT of the curves with 32-512 $\left(N_{t}\right)$ time points over 100-15000 $\left(N_{v}\right)$ voxels is shown in figure 5. As expected, the computation time per curve increased with the number of time points, but deceased with the number of curves that can be computed in parallel for both methods. However, the BlockFFT was 40-60\% faster than the CUFFT, due to the fact that the Block-FFT ignores the computation of the zero-padded points in the curves and applies the complex conjugates in frequency components of a real signal FT. The RMSE of a curve transformed by FT and followed by inverse FT was 2.33E-14 by the Block-FFT, compared to 2.46E-16 and $1.11 \mathrm{E}-16$ by the CUFFT and CPU GSL FFT, respectively. The small non-significant discrepancy of the RMSEs between the Block-FFT and CUFFT might be due to the numerical implementation of $\pi$ in the Fourier transformation.

To compute liver perfusion over a large number of voxels, multiple blocks run estimations simultaneously for multiple voxels on the GPU. Table 2 shows the performance of the GPU computation on the simulated DCE data. The computation time per voxel by GPU substantially decreased with the number of voxels due to parallel computation, and was much faster than by the C-CPU method except for only one voxel computed on the GPU $\left(N_{v}=1\right)$. For the DCE data with $32,64,128,256,320$ or 448 time points and 10000 voxels, the computation speeds by the GPU were 34, 32, 30, 28, 29 and 31 times faster than by the C-CPU method, respectively. Table 2 also provides $q \times p$ decompositions of 32,64, 128, 256, 320 and 448 time points, in which $N_{t}(=q p)$ were selected as close to the number of DCE imaging time 


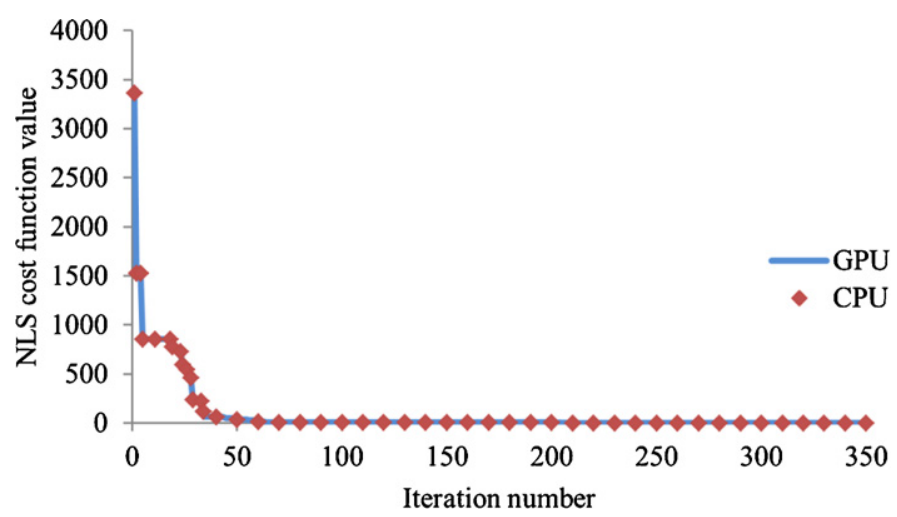

Figure 6. Convergence behaviors of the Simplex minimization implemented in the GPU and CCPU methods. Both methods have the exactly same convergence behavior, which is also the same as the one obtained from the Matlab-CPU method.

Table 2. Computation times of liver perfusion by GPU and C-CPU.

\begin{tabular}{|c|c|c|c|c|c|c|c|c|c|c|}
\hline \multicolumn{3}{|c|}{ Number of time points } & \multicolumn{7}{|c|}{ GPU time per voxel (msec) } & \multirow{2}{*}{$\begin{array}{l}\text { CPU time } \\
\text { per voxel } \\
(\mathrm{msec})\end{array}$} \\
\hline$N_{t}$ & $\mathrm{q}$ & $\times \mathrm{p}$ & $1^{\mathrm{a}}$ & 10 & 50 & 100 & 1000 & 5000 & 10000 & \\
\hline 32 & & $\times 16$ & 25.49 & 2.59 & 0.57 & 0.39 & 0.32 & 0.31 & 0.30 & 10.36 \\
\hline 64 & & $\times 32$ & 27.60 & 2.84 & 1.16 & 1.03 & 0.65 & 0.61 & 0.61 & 19.76 \\
\hline 128 & & $\times 64$ & 32.06 & 3.22 & 2.17 & 1.97 & 1.40 & 1.32 & 1.31 & 39.11 \\
\hline 256 & 4 & $\times 64$ & 41.85 & 4.19 & 3.31 & 3.30 & 2.96 & 2.95 & 2.94 & 82.48 \\
\hline 320 & 5 & $\times 64$ & 47.78 & 4.82 & 3.80 & 3.81 & 3.41 & 3.38 & 3.39 & 99.98 \\
\hline 448 & 7 & $\times 64$ & 60.92 & 6.10 & 4.84 & 4.83 & 4.34 & 4.31 & 4.31 & 136.71 \\
\hline
\end{tabular}

a The row is the number of voxels computed in parallel by the GPU method.

points as possible and $q$ was as small as possible but greater than 1. For the DCE data with the number of time points different from the examples in table 2, the dynamic data can be zero-padded to have a curve length the same as one of these cases.

The convergent behavior of the Simplex minimization for liver perfusion computation implemented on GPU was evaluated and compared to C-CPU and Matlab-CPU computations. Figure 6 shows the GPU method, CPU computations implemented with C and Matlab followed the exact same convergence course and were terminated after the same number of iterations for the given termination criteria when initiated by the same simplex.

The accuracy and stability of the GPU-based liver perfusion quantification under the influence of noise were evaluated. Table 3 shows the means and standard deviations of the parameters estimated from the 1000 simulated data by the GPU, C-CPU and Matlab-CPU methods. For the simulated DCE data with CNRs of 10 to 100, there were no differences in the means and standard deviations of the perfusion estimates among the three methods.

\subsection{Experimental patient study}

The volumetric hepatic perfusion parameters maps of the patients were estimated from DCE MRI by using the GPU and C-CPU computations. Figure 7 shows examples of slices of the liver perfusion parameters, estimated by the GPU method, in a patient. The means of the absolute differences of perfusion parameters over all the voxels in the liver between the GPU 
Table 3. Means and standard deviations of perfusion parameters estimated by the GPU, C-CPU and Matlab-CPU methods.

\begin{tabular}{|c|c|c|c|c|c|c|c|c|c|}
\hline \multirow[b]{2}{*}{ CNR } & \multicolumn{3}{|c|}{ GPU computation $(\mathrm{ml} / 100 \mathrm{~g} / \mathrm{min})$} & \multicolumn{3}{|c|}{ C-CPU estimation $(\mathrm{ml} / 100 \mathrm{~g} / \mathrm{min})$} & \multicolumn{3}{|c|}{ Matlab-CPU estimation $(\mathrm{ml} / 100 \mathrm{~g} / \mathrm{min})$} \\
\hline & $k_{l}$ & $k_{a}$ & $k_{p}$ & $k_{l}$ & $k_{a}$ & $k_{p}$ & $k_{l}$ & $k_{a}$ & $k_{p}$ \\
\hline$\infty^{\mathrm{a}}$ & 400.0 & 20.0 & 100.0 & 400.0 & 20.0 & 100.0 & 400.0 & 20.0 & 100.0 \\
\hline 100 & $400.1 \pm 7.1$ & $19.6 \pm 1.3$ & $100.4 \pm 1.6$ & $400.1 \pm 7.1$ & $19.6 \pm 1.3$ & $100.4 \pm 1.6$ & $400.1 \pm 7.1$ & $19.6 \pm 1.3$ & $100.4 \pm 1.6$ \\
\hline 90 & $399.7 \pm 13.2$ & $19.5 \pm 1.8$ & $99.2 \pm 3.0$ & $399.7 \pm 13.2$ & $19.5 \pm 1.8$ & $99.2 \pm 3.0$ & $399.7 \pm 13.2$ & $19.5 \pm 1.8$ & $99.2 \pm 3.0$ \\
\hline 80 & $398.3 \pm 19.1$ & $19.5 \pm 2.5$ & $100.0 \pm 4.6$ & $398.3 \pm 19.1$ & $19.5 \pm 2.5$ & $100.0 \pm 4.6$ & $398.3 \pm 19.1$ & $19.5 \pm 2.5$ & $100.0 \pm 4.6$ \\
\hline 70 & $398.2 \pm 25.7$ & $19.1 \pm 2.9$ & $100.4 \pm 6.5$ & $398.2 \pm 25.7$ & $19.1 \pm 2.9$ & $100.4 \pm 6.5$ & $398.2 \pm 25.7$ & $19.1 \pm 2.9$ & $100.4 \pm 6.5$ \\
\hline 60 & $399.1 \pm 29.4$ & $19.4 \pm 3.5$ & $100.3 \pm 7.5$ & $399.1 \pm 29.4$ & $19.4 \pm 3.5$ & $100.3 \pm 7.5$ & $399.1 \pm 29.4$ & $19.4 \pm 3.5$ & $100.3 \pm 7.5$ \\
\hline 50 & $398.9 \pm 32.6$ & $19.2 \pm 3.9$ & $99.1 \pm 8.3$ & $398.9 \pm 32.6$ & $19.2 \pm 3.9$ & $99.1 \pm 8.3$ & $398.9 \pm 32.6$ & $19.2 \pm 3.9$ & $99.1 \pm 8.3$ \\
\hline 40 & $398.9 \pm 40.7$ & $19.3 \pm 4.3$ & $99.9 \pm 10.6$ & $398.9 \pm 40.7$ & $19.3 \pm 4.3$ & $99.9 \pm 10.6$ & $398.9 \pm 40.7$ & $19.3 \pm 4.3$ & $99.9 \pm 10.6$ \\
\hline 30 & $400.0 \pm 49.2$ & $19.2 \pm 5.0$ & $101.0 \pm 12.3$ & $400.0 \pm 49.2$ & $19.2 \pm 5.0$ & $101.0 \pm 12.3$ & $400.0 \pm 49.2$ & $19.2 \pm 5.0$ & $101.0 \pm 12.3$ \\
\hline 20 & $401.9 \pm 54.4$ & $19.3 \pm 5.2$ & $101.3 \pm 13.7$ & $401.9 \pm 54.4$ & $19.3 \pm 5.2$ & $101.3 \pm 13.7$ & $401.9 \pm 54.4$ & $19.3 \pm 5.2$ & $101.3 \pm 13.7$ \\
\hline 10 & $398.4 \pm 59.3$ & $19.4 \pm 5.5$ & $99.7 \pm 15.6$ & $398.4 \pm 59.3$ & $19.4 \pm 5.5$ & $99.7 \pm 15.6$ & $398.4 \pm 59.3$ & $19.4 \pm 5.5$ & $99.7 \pm 15.6$ \\
\hline
\end{tabular}




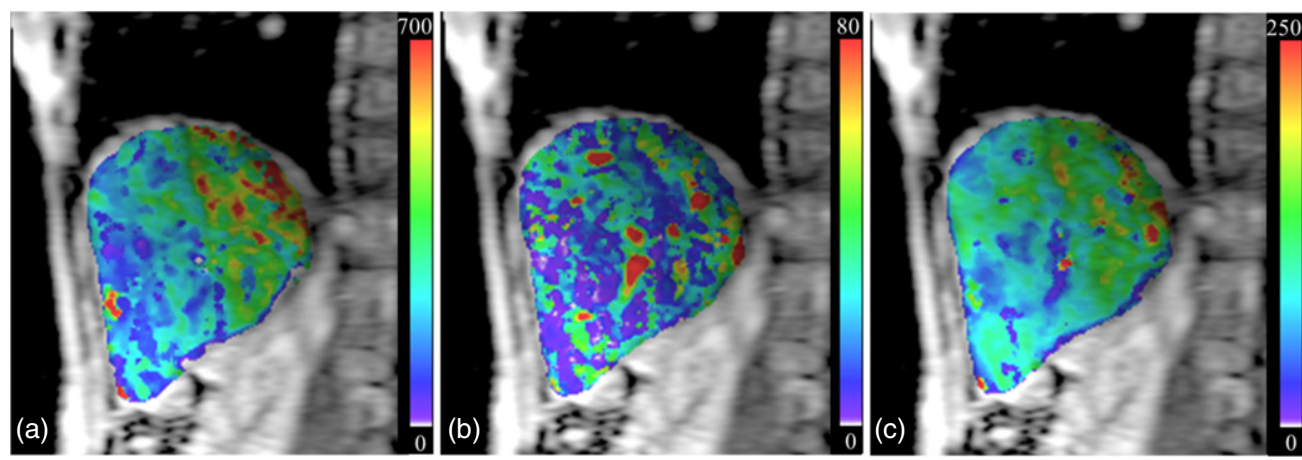

Figure 7. Hepatic perfusion maps of a liver slice estimated by the GPU computation: (a) estimated $k_{l}$ map; (b) estimated $k_{a}$ map; (c) estimated $k_{p}$ map. The perfusion parameters are in a unit of $\mathrm{ml} / 100 \mathrm{~g} / \mathrm{min}$

Table 4. The absolute differences of perfusion parameters between the GPU and C-CPU computations of all liver voxels.

\begin{tabular}{llll}
\hline & Patient 1 & Patient 2 & Patient 3 \\
\hline$k_{\mathrm{l}}$ & $2.4 \mathrm{E}-7 \pm 3.4 \mathrm{E}-6$ & $1.5 \mathrm{E}-7 \pm 2.6 \mathrm{E}-6$ & $1.2 \mathrm{E}-7 \pm 2.3 \mathrm{E}-6$ \\
$k_{a}$ & $1.7 \mathrm{E}-7 \pm 2.3 \mathrm{E}-5$ & $1.0 \mathrm{E}-7 \pm 8.3 \mathrm{E}-6$ & $6.1 \mathrm{E}-8 \pm 2.1 \mathrm{E}-6$ \\
$k_{p}$ & $2.4 \mathrm{E}-7 \pm 2.2 \mathrm{E}-5$ & $1.3 \mathrm{E}-7 \pm 5.8 \mathrm{E}-6$ & $7.7 \mathrm{E}-8 \pm 1.8 \mathrm{E}-6$ \\
\hline
\end{tabular}

Table 5. Total computation times for the voxelwise hepatic perfusion quantifications in three patients using the GPU and C-CPU computations.

\begin{tabular}{llllll}
\hline & $\begin{array}{l}\text { Voxel } \\
\text { number }\end{array}$ & $\begin{array}{l}\text { 1 GPU } \\
(\mathrm{min})\end{array}$ & $\begin{array}{l}\text { 2 GPUs } \\
(\mathrm{min})\end{array}$ & $\begin{array}{l}3 \text { GPUs } \\
(\mathrm{min})\end{array}$ & $\begin{array}{l}\text { CPU } \\
(\mathrm{min})\end{array}$ \\
\hline Patient 1 & 576556 & 2.31 & 1.27 & 0.83 & 103.01 \\
Patient 2 & 663980 & 2.63 & 1.44 & 0.95 & 117.08 \\
Patient 3 & 638728 & 2.73 & 1.50 & 0.98 & 116.35 \\
\hline
\end{tabular}

and C-CPU computations were in the order of magnitude from $10^{-4}$ to $10^{-5}$ in all three patients (table 4).

Likewise, showing that the GPU and C-CPU produced similar accuracy in liver perfusion quantification, the computation times per voxel by GPU and C-CPU were 0.25 and $10.74 \mathrm{msec}$, respectively. Table 5 shows the total times of the GPU and CPU perfusion quantifications in the whole livers of the three patients. For a liver with approximately 600000 voxels, the voxelwise perfusion computation lasted approximately $2 \mathrm{~h}$ by C-CPU, $2.5 \mathrm{~min}$ by single GPU, and less than 1 min by 3 GPUs.

\section{Discussion and conclusions}

In this paper, we developed a CUDA parallel computation method on an NVIDIA Tesla C2050 GPU to improve the speed of voxelwise hepatic perfusion quantification from DCE images. The parallel computations are not only executed over multiple voxels, but also over multiple time points in the cost function calculation. In addition, an efficient block-based 
FFT algorithm was implemented to convolve two time curves in the Fourier domain. Overall, we were able to achieve an approximately 30-fold improvement in the computation speed using a single GPU card, compared with using a CPU without compromise of the accuracy of the estimated parameters. The computation can be further accelerated if multiple GPUs are utilized. The parallel programming techniques reported here quell the longstanding concern about the computation time for voxelwise quantification of the hepatic perfusion over the whole liver in a clinical setting. Also, the framework as well as the components of the CUDA programming implementations, e.g., block-based FFT and parallel computation for the cost function over time points, can be generalized for other pharmacokinetic modeling of DCE data to substantially accelerate perfusion estimation in other organs.

GPU is a dedicated device for parallel computation. To achieve the best performance of the GPU computation, the first objective of the CUDA programming is to maximally parallelize the computation. Using the block-thread hierarchy in CUDA, perfusion parameters of multiple voxels are computed independently across multiple blocks, and Simplex minimization of one voxel is executed on a block with a parallel calculation of cost function values over multiple time points. Furthermore, individual convergence behavior of the Simplex optimization (figure 6) is allowed on each block to accommodate the different perfusion characteristics at a voxel.

We implement a Block-FFT for the Fourier domain convolution of two time curves. The Block-FFT is designed to run on a block for an arbitrary length of the time curve. By arranging a curve into a $q \times p$ table with $q>1$, the memory allocations for frequency components from row $q+1$ to $2 q-1$ in the frequency table can be omitted (figure 2). This reduction of the memory usage in the Block-FFT makes it possible to store the data on the limited shared memory for fast access. However, if the shared memory in an MP is not enough for any dataset, the frequency component table can be allocated on the device memory but with a tradeoff for slow device memory access. In the CUDA program, a block is configured to $(q+1) \times p$ threads to match the dimensions of the time or frequency table. Note that this configuration is also limited by the maximal number of threads within a block. Our implementation allows perfusion quantification from DCE data with 448 time points or less on the Tesla C2050 GPU. The maximal number of threads in one block for the GPU card is 1024, which is quite generous for DCE data acquired in a clinical setting.

We report a method to accelerate voxel-by-voxel liver perfusion computation from volumetric DCE imaging by using the dual-input single-compartment model. The hepatic perfusion consists of two phases, i.e. the arterial and portal venous perfusion. Although a single vascular input model is popularly used for characterizing the vascular behavior of a highly arterialized tumor, the dual-input single-compartmental model is more reasonable in representing the underlying physiology of hepatic perfusion, particularly in situations where the clinical and scientific interests are not limited to the tumor, e.g., cirrhosis, and drug and radiation effects on the liver parenchyma (Hashimoto et al 2006, Leggett et al 1997, Van Beers et al 2001). The latter studies (Cao et al 2007, 2012) show it is not only that portal venous perfusion needs to be considered but also it is necessary to map the whole liver perfusion voxel-by-voxel. Finally, although our method is designed to accelerate the perfusion quantification using the conventional hepatic dual-input single compartment model, the GPUbased parallel computation framework can be applied to other pharmacokinetic models to speed up the computation (Tofts 1997, Koh et al 2008) since the convolution and optimization of a least-squares cost function are the common computations in these models.

In conclusion, the GPU method greatly speeds up the computation of voxelwise hepatic perfusion from DCE data. The method may make volumetric perfusion evaluation practical in clinical applications. The quantitative liver perfusion imaging may improve 
detection of liver disease and assessment of tissue therapeutic response beyond region-based quantifications.

\section{Acknowledgments}

This work was supported in part by NIH P01 CA59827, RO1 CA132834 and RO1 NS064973. We would like to thank Dr James Balter from the Department of Radiation Oncology of the University of Michigan for providing the machine with GPU cards for this study.

\section{References}

Cao Y, Alspaugh J, Shen Z, Balter J M, Lawrence T S and Ten Haken R K 2006 A practical approach for quantitative estimates of voxel-by-voxel liver perfusion using DCE imaging and a compartmental model Med. Phys. 33 3057-62

Cao Y, Platt J F, Francis I R, Balter J M, Pan C, Normolle D, Ben-Josef E, Haken R K and Lawrence T S 2007 The prediction of radiation-induced liver dysfunction using a local dose and regional venous perfusion model Med. Phys. 34 604-12

Cao Y et al 2012 Prediction of liver function by using magnetic resonance-based portal venous perfusion imaging Int. J. Radiat. Oncol. Biol. Phys. (at press) http://dx.doi.org/10.1016/j.ijrobp.2012.02.037

Chiandussi L, Greco F, Sardi G, Vaccarino A, Ferraris C M and Curti B 1968 Estimation of hepatic arterial and portal venous blood flow by direct catheterization of the vena porta through the umbilical cord in man. Preliminary results Acta Hepatosplenol. 15 166-71

Daniel H 2005 Stream reduction operations for GPGPU applications GPU Gems 2 573-89

Dawson P 2007 Liver perfusion measurements Br. J. Radiol. 801024

Duhamel P and Vetterli M 1990 Fast Fourier transforms: a tutorial review and a state of the art Signal Process. 19 259-99

Feng D, Huang S C, Wang Z Z and Ho D 1996 An unbiased parametric imaging algorithm for nonuniformly sampled biomedical system parameter estimation IEEE Trans. Med. Imaging 15 512-8

Galassi M, Davies J, Theiler J, Gough B, Jungman G, Alken P, Booth M and Rossi F 2009 GNU Scientific Library Reference Manual 3rd edn (New York: Network Theory Ltd)

Goetti R, Reiner C S, Knuth A, Klotz E, Stenner F, Samaras P and Alkadhi H 2012 Quantitative perfusion analysis of malignant liver tumors: dynamic computed tomography and contrast-enhanced ultrasound Invest. Radiol. 47 18-24

Hagiwara M, Rusinek H, Lee V S, Losada M, Bannan M A, Krinsky G A and Taouli B 2008 Advanced liver fibrosis: diagnosis with 3D whole-liver perfusion MR imaging-initial experience Radiology 246 926-34

Hashimoto K et al 2006 Assessment of the severity of liver disease and fibrotic change: the usefulness of hepatic CT perfusion imaging Oncol. Rep. 16 677-83

Jia X, Gu X, Graves Y J, Folkerts M and Jiang S B 2011 GPU-based fast Monte Carlo simulation for radiotherapy dose calculation Phys. Med. Biol. 56 7017-31

Koh T S, Thng C H, Lee P S, Hartono S, Rumpel H, Goh B C and Bisdas S 2008 Hepatic metastases: in vivo assessment of perfusion parameters at dynamic contrast-enhanced MR imaging with dual-input two-compartment tracer kinetics model Radiology 249 307-20

Lagarias J C, Reeds J A, Wright M H and Wright P E 1998 Convergence properties of the Nelder-Mead simplex method in low dimensions SIAM J. Optim. 9 112-47

Leggett D A, Kelley B B, Bunce I H and Miles K A 1997 Colorectal cancer: diagnostic potential of CT measurements of hepatic perfusion and implications for contrast enhancement protocols Radiology 205 716-20

Materne R, Smith A M, Peeters F, Dehoux J P, Keyeux A, Horsmans Y and Van Beers B E 2002 Assessment of hepatic perfusion parameters with dynamic MRI Magn. Reson. Med. 47 135-42

Miyazaki S, Murase K, Yoshikawa T, Morimoto S, Ohno Y and Sugimura K 2008 A quantitative method for estimating hepatic blood flow using a dual-input single-compartment model Br. J. Radiol. 81 790-800

Moreland K and Angel E 2003 The FFT on a GPU Proc. ACM Conf. on Graphics Hardware pp 112-9

Murase K, Miyazaki S and Yang X 2007 An efficient method for calculating kinetic parameters in a dual-input single-compartment model Br. J. Radiol. $80371-5$

Nelder J A and Mead R 1965 A simplex method for function minimization Comput. J. 7 308-13

NVIDIA 2010 CUDA C programming guide (Santa Clara, CA: NVIDIA Corporation) 
Oppenheim A V, Schafer R W and Buck J R 1999 Discrete-time Signal Processing (Upper Saddle River, NJ: PrenticeHall)

Owens J D, Houston M, Luebke D, Green S, Stone J E and Phillips J C 2008 GPU Computing Proc. IEEE 96 879-99

Pandharipande P V, Krinsky G A, Rusinek H and Lee V S 2005 Perfusion imaging of the liver: current challenges and future goals Radiology 234 661-73

Pratx G and Xing L 2011 GPU computing in medical physics: a review Med. Phys. 38 2685-97

Press W H, Teukolsky S A, Vetterling W T and Flannery B P 2007 Numerical Recipes 3rd edition: the Art of Scientific Computing (New York: Cambridge University Press)

Thng C H, Koh T S, Collins D J and Koh D M 2010 Perfusion magnetic resonance imaging of the liver World J. Gastroenterol. 16 1598-609

Tofts P S 1997 Modeling tracer kinetics in dynamic Gd-DTPA MR imaging J. Magn. Reson. Imaging $791-101$

Van Beers B E, Leconte I, Materne R, Smith A M, Jamart J and Horsmans Y 2001 Hepatic perfusion parameters in chronic liver disease: dynamic CT measurements correlated with disease severity AJR Am. J. Roentgenol. $176667-73$ 